\title{
Cognitive Behavioral Therapy for Tinnitus: Evidence and Efficacy
}

\author{
Hyung Jin Jun ${ }^{1}$ and Moo Kyun Park ${ }^{2}$ \\ ${ }^{1}$ Department of Otolaryngology-Head and Neck Surgery, Korea University College of Medicine, Seoul, \\ ${ }^{2}$ Department of Otolaryngology-Head and Neck Surgery, Bucheon Hospital, Soonchunhyang University College of Medicine, \\ Bucheon, Korea
}

Received November 16, 2013

Revised November 20, 2013

Accepted November 21, 2013
Tinnitus is defined as auditory perception without external sound. There is currently no cure for tinnitus. Cognitive behavioral therapy (CBT) is a tinnitus treatment that addresses the affected individual's reaction to tinnitus. It aims not to eliminate auditory perception as sound but to reduce or correct one's negative response to tinnitus. CBT identifies negative automatic thought and then evaluates its validity with the patient. It also aims to change negative automatic thought to more positive and realistic thought. In this way, tinnitus sufferers can function well despite the presence of tinnitus. Many studies have supported the efficacy of CBT for treating tinnitus. A meta-analysis of CBT for tinnitus also concluded that CBT is effective in treating tinnitus. Thus, CBT is considered a good option for treating tinnitus. We herein discuss the use of CBT for tinnitus with a literature review.

Korean J Audiol 2013;17:101-104

Tel +82-32-621-5054

Fax $+82-32-621-5440$

E-mail aseptic@schmc.ac.kr

\section{Introduction}

Tinnitus is defined as auditory perception without external sound stimulation. Tinnitus is known to affect between $5 \%$ and $15 \%$ of the population, ${ }^{1)}$ and $1 \%$ of the population has deliberating tinnitus. ${ }^{2)}$ Tinnitus can affect almost all aspects of life. Additionally, it results in a high need for medical care.

A variety of treatments have been introduced for tinnitus, such as sound treatment, tinnitus retraining therapy, and medications such as anxiolytics and antidepressants. However, none of these treatments has proven to be a cure for tinnitus. Furthermore, these treatments do not seem to be very effective or satisfactory for patients. Tinnitus treatment can be categorized into two types. One is the treatment of tinnitus perception, whose goal is to eliminate or reduce tinnitus perception. The other is treatment of the response to tinnitus, which focuses on the effect of tinnitus on one's life. These treatments aim to reduce distress and anxiety caused by tinnitus. Examples in-

This is an Open Access article distributed under the terms of the Creative Commons Attribution Non-Commercial License (http://creativecommons. org/licenses/by-nc/3.0/) which permits unrestricted non-commercial use, distribution, and reproduction in any medium, provided the original work is properly cited. clude cognitive behavioral therapy (CBT), commitment and acceptance therapy, and mindfulness. ${ }^{3-5)}$

CBT has been studied by many researchers and was originally developed by Beck. ${ }^{6)} \mathrm{CBT}$ is a time-limited, structured therapy. The therapist usually performs eight to 24 weekly CBT sessions. Beck first applied CBT to depression, but rationales have also been provided for its application in treating insomnia, chronic pain, and anxiety. ${ }^{7}$ The main concept of CBT is that negative and unrealistic cognitions (thoughts and beliefs) cause maladaptive behavior. By challenging (correcting) these negative cognitions, the patient can have more positive and realistic thoughts.

CBT for tinnitus focuses on reducing the distress and handicap induced by tinnitus. The goal of CBT is not to reduce the acoustic features of the condition, such as loudness or pitch, but to help patients who face specific difficulties in everyday situations by constructing more positive and realistic thoughts about their situation. CBT typically comprises both cognitive and behavioral therapy including cognitive restructuring, relaxation training, imagery techniques, and exposure to difficult situations. $^{8)}$ 
Table 1. Significant tinnitus studies and their outcomes

\begin{tabular}{|c|c|c|c|}
\hline Study & Design & Positive findings & Negative findings \\
\hline$\overline{\text { Sweetow }}{ }^{16)}$ & Case series & Introduction to CBT & \\
\hline Kröner-Herwig, et al. ${ }^{23)}$ & Case-control study & $\begin{array}{l}\text { More satisfaction with training } \\
\text { than with yoga }\end{array}$ & \\
\hline Henry and Wilson ${ }^{20)}$ & Case-control study & $\begin{array}{l}\text { Distress, handicaps associated } \\
\text { with tinnitus; engagement } \\
\text { in dysfunctional cognition }\end{array}$ & $\begin{array}{l}\text { No effect on depression or } \\
\text { subjective loudness }\end{array}$ \\
\hline Andersson, et al. ${ }^{26)}$ & Controlled trial & Effectiveness in the elderly & \\
\hline Rief, et al. ${ }^{21)}$ & Randomized clinical trial & Emotional distress & $\begin{array}{l}\text { Reactivity of head muscles at the } \\
\text { beginning predicted significant } \\
\text { treatment effects }\end{array}$ \\
\hline Kaldo, et al. ${ }^{24)}$ & Randomized clinical trial & $\begin{array}{l}\text { Effectiveness of CBT-based } \\
\text { self-help book }\end{array}$ & \\
\hline Weise, et al. ${ }^{22)}$ & Randomized clinical trial & $\begin{array}{l}\text { Tinnitus annoyance, diary } \\
\text { ratings of loudness, feelings } \\
\text { of controllability }\end{array}$ & \\
\hline Andersson and Lyttkens ${ }^{19)}$ & Meta-analysis & $\begin{array}{l}\text { Strong effect on tinnitus } \\
\text { annoyance, some effect } \\
\text { on tinnitus loudness }\end{array}$ & $\begin{array}{l}\text { Lower effect on negative affect } \\
\text { and sleep problems }\end{array}$ \\
\hline Hesser, et al. ${ }^{17)}$ & Meta-analysis & $\begin{array}{l}\text { Tinnitus distress } \\
\text { Effective in the long term }\end{array}$ & \\
\hline Martinez-Devesa, et al. ${ }^{18)}$ & Cochrane review & Depression score, quality of life & No effect on subjective loudness \\
\hline
\end{tabular}

CBT: cognitive behavioral therapy

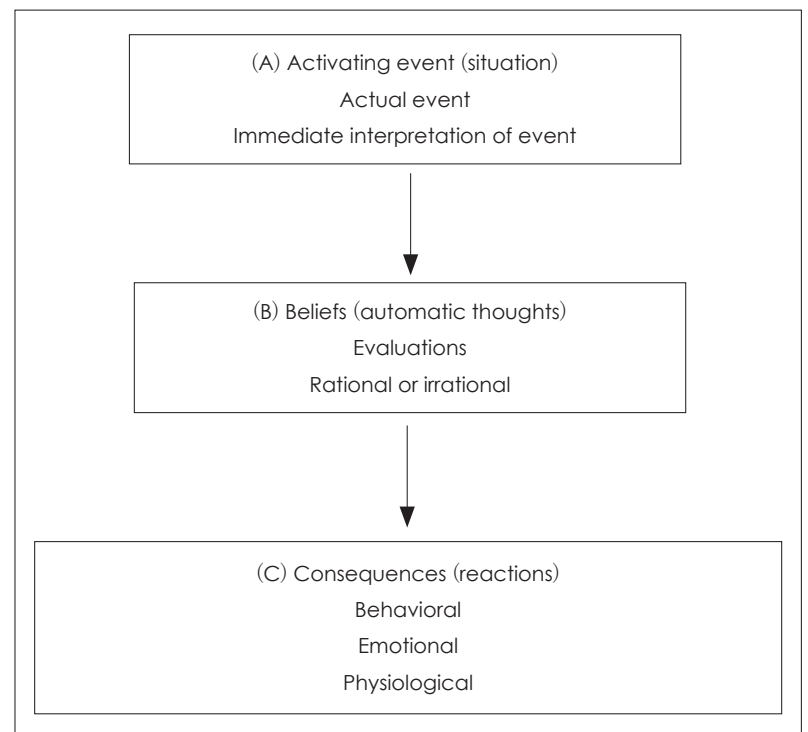

Fig. 1. A-B-C model of cognitive behavioral therapy.

\section{Description of CBT Principles}

\section{Cognitive model}

The principle of CBT is identifying a negative thought about a specific situation and then changing this negative thought to a more realistic and positive thought. The general framework of CBT is known as the A-B-C model (Fig. 1). One of the most important procedures in CBT is the identification of a belief or automatic thought. An automatic thought is one that arises spontaneously in response to a specific situation. Beck found that, in depressed patients, unrealistic and negative thoughts

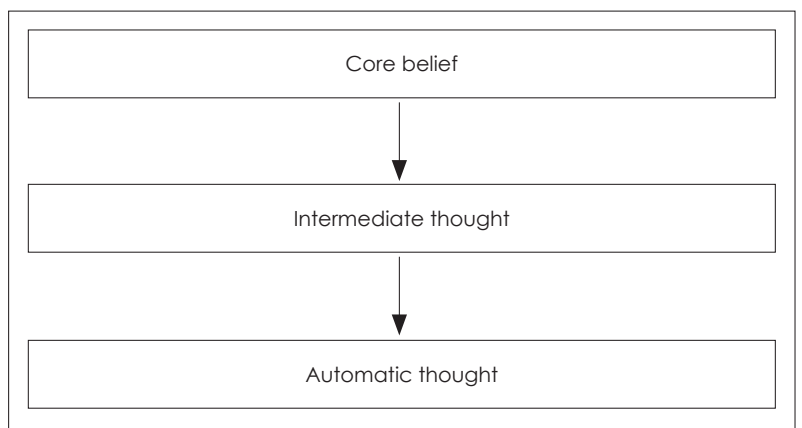

Fig. 2. Cognitive model.

underlay depression. Such thoughts arise automatically, so affected individuals tend to accept them as true. ${ }^{9)}$

Cognition refers to one's thoughts and beliefs. Cognitive models tell us that cognition comprises automatic thought, intermediate thought, and core beliefs. Core beliefs usually develop in childhood and are more fundamental, defining the way one interprets the outside world. Intermediate thoughts are similar to rules or assumptions. Automatic thoughts arise spontaneously in response to specific situations. Because core beliefs are fundamental, they also influence automatic thoughts (Fig. 2). ${ }^{9)}$

Like depressive patients, some tinnitus patients have unrealistic and negative thoughts about tinnitus. CBT aims to identify these negative automatic thoughts, evaluate their validity, and replace them with more realistic and positive thoughts. This procedure is referred to as cognitive restructuring. 


\section{Challenging automatic thought}

Patients have a tendency to view their automatic thoughts as truth. Because automatic thoughts are, literally, automatic, they are considered to "pop up" in one's mind. In CBT, the clinician and patient work together to frame the automatic negative thought as a hypothesis that may or may not be true. In a process called empirical collaboration, the clinician and patient evaluate the patient's negative thoughts together. Gathering proof to support as well as proof against the automatic thoughts provides a chance to evaluate the validity of the those thoughts. ${ }^{9,10)}$ After evaluating the negative automatic thoughts, the clinician offers more positive and realistic thoughts to replace them. This procedure (identifying automatic thoughts and evaluating their validity) should be performed not only during counseling sessions but also between sessions. The patient's active participation is essential, which is why homework is important.

\section{Treatment rationale}

Tinnitus may be conceived as a failure to adapt to a stimulus. ${ }^{11)}$ Habituation is defined as insensitivity due to exposure to a repeated stimulus. Tinnitus can be interpreted as sensitivity to an internal sound. Cognition refers to thought and belief. Cognition may have a role in a patient's reactions to tinnitus, and a mechanism of tinnitus distress has been suggested. First, the tinnitus is evaluated as harmful, which induces anxiety. The duration of tinnitus is unpredictable, and the patient feels a loss of control over the condition. ${ }^{12)}$ Hallam, et al. ${ }^{13-15)}$ pointed out that tinnitus may result from failed habituation and growing attention to a signal. Jastreboff suggested a neurophysiologic model of tinnitus. In this model, the perception of tinnitus as distressing is related to emotional factors. CBT aims to change one's negative thoughts to more positive ones; the effectiveness of CBT for patients with tinnitus may lie in changing unhealthy thoughts about their own tinnitus. In this sense, CBT may represent a neurophysiological model of tinnitus.

\section{Evidence of the effectiveness of CBT for tinnitus}

Although cognitive therapy was developed as a psychological treatment for pain, depression, anxiety, and other psychological conditions, it also can apply to tinnitus. If tinnitus causes a specific behavior, behavioral therapy using conditioning principles can be applied to the treatment of that behavior. Cognitive therapy cannot cure or eliminate tinnitus; however, it is one of the most widely used therapy methods for tinnitus. Since Hallam and Sweetow first introduced CBT for tinnitus in the 1980s, many studies have demonstrated the value of this approach (Table 1). ${ }^{16)}$

Results of meta-analytic reviews have demonstrated that
CBT is effective for alleviating the annoyance caused by tinnitus and has some positive effects on other emotional symptoms. However, they found little effect on tinnitus loudness. ${ }^{17-19)}$

Several randomized controlled studies have demonstrated various positive effects of CBT on tinnitus. Henry and Wilson $^{20)}$ demonstrated that combined cognitive/educational intervention significantly reduced the distress and handicap associated with tinnitus as well as the tendency to engage in dysfunctional cognition compared with subjects who received education alone. However, these authors did not observe significant effects on measures of depression, locus of control, or daily ratings of subjective loudness, noticeability, or bothersomeness of the tinnitus. Furthermore, the benefits of these treatments had disappeared by the 12-month follow-up. Another study also found improvement in tinnitus annoyance and a significant reduction in emotional distress in the CBT treatment group. $^{21)}$

CBT using biofeedback has also resulted in clear improvements in tinnitus annoyance, diary ratings of loudness, and feelings of controllability. Furthermore, changes in coping cognition and depressive symptoms were found. Improvements were maintained over a 6-month follow-up period, during which medium to large effect sizes were observed. ${ }^{22)}$

Kröner-Herwig, et al. ${ }^{23)}$ reported that the CBT group was more satisfied than the control or yoga treatment group. Kaldo, et al. ${ }^{24)}$ demonstrated that reduction in tinnitus distress due to a CBT-based self-help book was comparable to that from formal CBT. Although the effect size was smaller than that of formal CBT, they thought that it was more cost-effective because of the shortage of CBT therapists. The results of CBT have been shown to be superior to minimal contact interventions using bedside education or music-supported relaxation, as well as to results for a wait-list control group. ${ }^{25)}$ Elderly patients also showed statistically significant reductions in tinnitus-related distress in a controlled trial of $\mathrm{CBT}^{26)}$

\section{Outcomes of CBT in a meta-analysis}

A meta-analysis by Hesser, et al. ${ }^{17)}$ demonstrated that CBT improved the mean effect sizes of tinnitus-specific measures. The average weighted pre-to-post effect size for the CBT group showed that these effects were maintained over time. They also found smaller but statistically significant effects of CBT on mood outcome measures.

In a Cochrane study in 2010, significant improvements in depression scores and quality of life were found. They also found that CBT had a positive effect on the management of tinnitus. However, they did not find evidence of a significant change in the subjective loudness of tinnitus. ${ }^{18)}$

Andersson and Lyttkens ${ }^{19)}$ demonstrated a moderate to strong 
effect on tinnitus annoyance in a meta-analytic review. They also found some effects on tinnitus loudness. However, CBT had less effect on negative affect and sleep problems.

\section{Conclusions}

CBT can be applied to tinnitus treatment. It does not have an effect on improving the acoustic characteristic of tinnitus, but it improves the response to tinnitus. Thus, CBT is a good treatment option for tinnitus.

\section{Acknowledgments}

This work was supported by the Soonchunhyang University Research Fund.

\section{REFERENCES}

1) Heller AJ. Classification and epidemiology of tinnitus. Otolaryngol Clin North Am 2003;36:239-48.

2) Axelsson A, Ringdahl A. Tinnitus--a study of its prevalence and characteristics. Br J Audiol 1989;23:53-62.

3) Andersson G. Psychological aspects of tinnitus and the application of cognitive-behavioral therapy. Clin Psychol Rev 2002;22:977-90.

4) Philippot P, Nef F, Clauw L, de Romrée M, Segal Z. A randomized controlled trial of mindfulness-based cognitive therapy for treating tinnitus. Clin Psychol Psychother 2012;19:411-9.

5) Westin VZ, Schulin M, Hesser H, Karlsson M, Noe RZ, Olofsson $\mathrm{U}$, et al. Acceptance and commitment therapy versus tinnitus retraining therapy in the treatment of tinnitus: a randomised controlled trial. Behav Res Ther 2011;49:737-47.

6) Beck AT, Rush AJ, Shaw BF, Emery G. Cognitive therapy of depression. New York: Guilford Press; 1979.

7) Hawton KE, Salkovskis PM, Kirk JE, Clark DM. Cognitive Behaviour Therapy for Psychiatric Problems: A Practical Guide. Oxford: Oxford University Press; 1989.

8) Greimel KV, Kröner-Herwig B. Cognitive behavioral treatment (CBT). In: Greimel KV, Kröner-Herwig B, editors. Textbook of tinnitus. New York: Springer;2011. p.557-61.

9) Beck JS. Cognitive behavior therapy: basics and beyond. 2nd ed. New York: Guilford Press;2011.

10) Thorn BE. Cognitive therapy for chronic pain: a step-by-step guide. New York: Guilford Press;2004.

11) Hallam RS, Rachman S, Hinchcliffe R. Psychological aspects of tin- nitus. In: Rachmen S, editor. Contributions to Medical Psychology. Vol 3. Oxford: Pergamon;1984. p.31-53.

12) Zenner HP, Pfister M, Birbaumer N. Tinnitus sensitization: sensory and psychophysiological aspects of a new pathway of acquired centralization of chronic tinnitus. Otol Neurotol 2006;27:1054-63.

13) Hallam RS, Jakes SC, Chambers C, Hinchcliffe R. A comparison of different methods for assessing the 'intensity' of tinnitus. Acta Otolaryngol 1985;99:501-8.

14) Hallam RS, Jakes SC, Hinchcliffe R. Cognitive variables in tinnitus annoyance. Br J Clin Psychol 1988;27(Pt 3):213-22.

15) Hallam RS, McKenna L, Shurlock L. Tinnitus impairs cognitive efficiency. Int J Audiol 2004;43:218-26.

16) Sweetow RW. Cognitive aspects of tinnitus patient management. Ear Hear 1986;7:390-6.

17) Hesser H, Weise C, Westin VZ, Andersson G. A systematic review and meta-analysis of randomized controlled trials of cognitive-behavioral therapy for tinnitus distress. Clin Psychol Rev 2011;31:54553.

18) Martinez-Devesa P, Perera R, Theodoulou M, Waddell A. Cognitive behavioural therapy for tinnitus. Cochrane Database Syst Rev 2010: CD005233.

19) Andersson G, Lyttkens L. A meta-analytic review of psychological treatments for tinnitus. Br J Audiol 1999;33:201-10.

20) Henry JL, Wilson PH. The Psychological Management of Tinnitus: Comparison of a Combined Cognitive Educational Program, Education Alone and a Waiting-List Control. Int Tinnitus J 1996;2:9-20.

21) Rief W, Weise C, Kley N, Martin A. Psychophysiologic treatment of chronic tinnitus: a randomized clinical trial. Psychosom Med 2005; 67:833-8.

22) Weise $C$, Heinecke $K$, Rief W. Biofeedback-based behavioral treatment for chronic tinnitus: results of a randomized controlled trial. J Consult Clin Psychol 2008;76:1046-57.

23) Kröner-Herwig B, Hebing G, van Rijn-Kalkmann U, Frenzel A, Schilkowsky G, Esser G. The management of chronic tinnitus-comparison of a cognitive-behavioural group training with yoga. J Psychosom Res 1995;39:153-65.

24) Kaldo V, Cars S, Rahnert M, Larsen HC, Andersson G. Use of a selfhelp book with weekly therapist contact to reduce tinnitus distress: a randomized controlled trial. J Psychosom Res 2007;63:195-202.

25) Kröner-Herwig B, Frenzel A, Fritsche G, Schilkowsky G, Esser G. The management of chronic tinnitus: comparison of an outpatient cognitive-behavioral group training to minimal-contact interventions. J Psychosom Res 2003;54:381-9.

26) Andersson G, Porsaeus D, Wiklund M, Kaldo V, Larsen HC. Treatment of tinnitus in the elderly: a controlled trial of cognitive behavior therapy. Int J Audiol 2005;44:671-5. 